\title{
Cultura ambiental y técnicas de enseñanza. El caso de una secundaria general de Cd. Victoria, Tamaulipas
}

Environmental Culture and Teaching Techniques. A Case of a Middle School in Cd. Victoria, Tamaulipas

Cultura ambiental e técnicas de ensino. $\mathrm{O}$ caso de um secundário geral do $\mathrm{Cd}$. Victoria, Tamaulipas

Rosa Delia Cervantes Castro

Universidad Autónoma de Tamaulipas, México rdcervantes@docentes.uat.edu.mx https://orcid.org/0000-0001-5214-9366

Xóchitl Gómez Cordero

Universidad Autónoma de Tamaulipas, México xgomez@docentes.uat.edu.mx https://orcid.org/0000-0002-5475-7944

Martín Dagoberto Olguín Villarreal Universidad Autónoma de Tamaulipas, México martinillo2691@hotmail.com https://orcid.org/0000-0002-2753-5756 


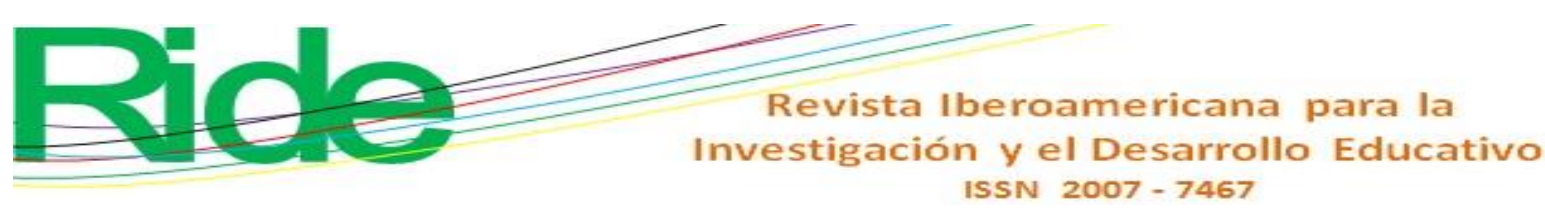

\section{Resumen}

La cultura ambiental actualmente es un tema relevante y prioritario alrededor del mundo. $\mathrm{Su}$ importancia recae en que, con una conciencia crítica, los recientes sucesos que afectan el medio ambiente pueden interrumpirse. En este sentido, es necesario enfocar la educación ambiental hacia la formación de valores y concientización para crear actitudes favorables con el planeta. En los programas de estudio de educación secundaria se incorpora este tema, y resulta interesante saber cómo se enseña por parte de los profesores.

Este estudio tuvo como objetivo principal analizar las técnicas de enseñanza que utiliza el docente y descubrir si estas permiten desarrollar un pensamiento crítico y de reflexión respecto al cuidado del ambiente en estudiantes de secundaria. Consecuentemente, se consideró prioritario explorar la manera en la que los alumnos conciben la educación ambiental que reciben en el aula, y de qué forma impacta en su conducta diaria.

La investigación fue de tipo cuantitativa-explicativa. Se encuestó a dos profesores de la asignatura de Ciencias I. Los principales resultados arrojaron que el camino mediante el cual el profesor los lleva a la reflexión es a través de técnicas como el debate dirigido. Esto les permite a los alumnos informarse sobre problemáticas, al igual que debatir y reflexionar sobre su forma de actuar. Asimismo, al preguntar sobre la forma en cómo el docente imparte la clase, los porcentajes apuntan a que, cuando los alumnos no cuentan con información clara, hay una explicación oportuna. Por lo tanto, los resultados sugieren que las técnicas que utilizan los docentes en la secundaria general permiten que los estudiantes desarrollen un pensamiento crítico sobre el cuidado del medio ambiente.

Palabras clave: cultura, educación ambiental, técnica de enseñanza.

\section{Abstract}

Nowadays, environmental culture is a relevant and priority issue all around the globe. Its relevance lays on the recent events that affect the environment and that could be prevented. Accordingly, it is necessary to focus environmental education towards the formation of values and raising awareness to create beneficial attitudes towards the planet. In middle school education study programs this topic is included; therefore, it is interesting to explore how teachers teach it.

The main objective of this study was to analyze the teaching techniques used by the middle school teacher to understand if they allow the development of a critical thinking and reflection on environmental care in middle school students. Therefore, we consider as a priority to explore the 


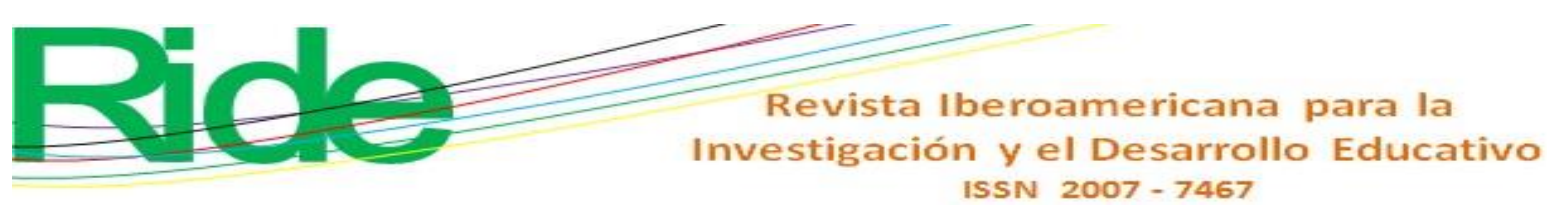

way in which students conceive the environmental education they receive in the classroom, and how it influences their daily behavior.

This research was quantitative-explanatory. Two professors of the science courses were surveyed. The main results show that the teacher leads them to reflection through techniques such as moderated discussions. This allows students to learn about problems, discuss their importance and reflect on their way of acting. In addition, when asking about the way in which the teacher teaches the class, the percentages suggest that when students do not have clear information, there is a timely explanation. Therefore, the results suggest that the techniques used by teachers in general middle school allow students to develop critical thinking about the care of the environment. Keywords: culture, environmental education, teaching techniques.

\section{Resumo}

Atualmente, a cultura ambiental é uma questão relevante e prioritária em todo o mundo. Sua importância reside no fato de que, com uma consciência crítica, eventos recentes que afetam o meio ambiente podem ser interrompidos. Nesse sentido, é necessário focar a educação ambiental na formação de valores e conscientização para criar atitudes favoráveis com o planeta. Esse tópico é incorporado aos programas de ensino médio e é interessante saber como é ensinado pelos professores.

O principal objetivo deste estudo foi analisar as técnicas de ensino utilizadas pelo professor e descobrir se elas permitem o desenvolvimento de pensamento crítico e reflexão sobre o cuidado com o meio ambiente em alunos do ensino médio. Consequentemente, foi considerado prioritário explorar a maneira pela qual os alunos concebem a educação ambiental que recebem na sala de aula e como isso afeta seu comportamento diário.

A pesquisa foi quantitativa-explicativa. Foram pesquisados dois professores da disciplina de Ciências I. Os principais resultados mostraram que o caminho pelo qual o professor os leva à reflexão é por meio de técnicas como o debate direcionado. Isso permite que os alunos aprendam sobre os problemas, além de discutir e refletir sobre sua maneira de agir. Além disso, ao perguntar sobre a maneira como o professor ensina a classe, as porcentagens sugerem que, quando os alunos não têm informações claras, há uma explicação oportuna. Portanto, os resultados sugerem que as técnicas utilizadas pelos professores no ensino médio em geral permitem que os alunos desenvolvam um pensamento crítico sobre o cuidado com o meio ambiente. 


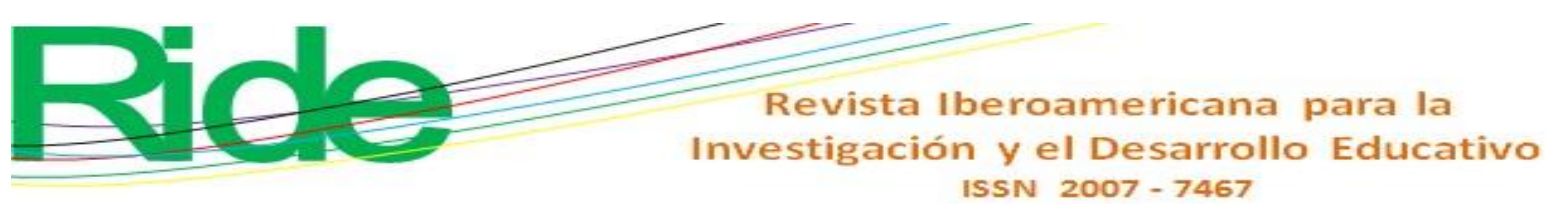

Palavras-chave: cultura, educação ambiental, técnica de ensino.

Fecha Recepción: Abril 2019

Fecha Aceptación: Septiembre 2019

\section{Introducción}

Los problemáticas ambientales que enfrentamos hoy en día son consecuencia del mal uso que le estamos dando a los recursos naturales que nos brinda el planeta. A tal grado que los daños son casi irreversibles. Sin embargo, contamos con una herramienta indispensable que necesitamos utilizar para afrontar este gran desafío: la educación ambiental. La educación ambiental busca ayudar a las personas y a los grupos sociales a que adquieran mayor sustentabilidad y conciencia del medio ambiente en general y de los problemas relacionados. $\mathrm{Y}$ al mismo pretende que adquieran una comprensión básica del medio ambiente en su totalidad. Es por ello por lo que, tal y como lo mencionan los programas conjuntos de la Organización de las Naciones Unidas para la Educación, la Ciencia y la Cultura [Unesco] (2018), es necesario fomentar la educación en materia de sostenibilidad del medio ambiente en la gestión del patrimonio.

La educación ambiental, mediante las recomendaciones de la Unesco (2018), difunde, a nivel local y mundial, diferentes tendencias con el fin de crear actitudes positivas para enfrentar un cambio que si bien parece muy lejano no es imposible, claro, con la ayuda de todos los seres humanos. Sin duda la crisis ecológica fue la causante de poner este tópico sobre las mesas de discusión y analizar los orígenes de los factores que intervienen en la destrucción del planeta Tierra.

La actual conceptualización social del desarrollo sostenible debe ser entendida como un proceso continuo y permanente que constituye una dimensión de la educación integral de todos los ciudadanos, orientada a la adquisición de conocimientos, desarrollo de hábitos, habilidades, capacidades y actitudes y en la formación de valores que fortalezcan las relaciones entre los seres humanos y la naturaleza (Secretaría de Educación Pública [SEP], 2011b).

En este sentido, se debe recurrir a la educación ambiental para desarrollar una cultura ambiental, utilizarla como una herramienta para la sociedad, y sustituir la visión que tenemos del concepto de cuidado ambiental, porque el equilibrio del planeta se ve amenazado por nuestra civilización. Es importante resaltar que hace muchos años la situación que se vive hoy en día respecto a los cambios ambientales era inimaginable, pero, debido a las profundas transformaciones que obligan a modernizar al hombre constantemente, estos cambios ya son, en muchos casos, irreversibles. 


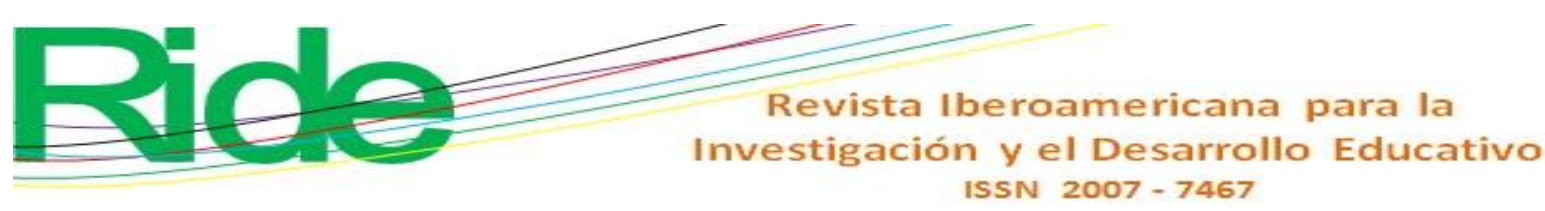

sociedad y la naturaleza, pero para esto es importante conocer el concepto de educación ambiental desde su concepción.

La educación ambiental, desde una perspectiva amplia, debe tener la capacidad de promover esquemas sistémicos acordes al desarrollo sociocultural en el pleno ambiental, como lo dice Ballesteros y Covarrubias (1997; citados en Maldonado, 2005). Además, debe de ser asumida como un proceso educativo integral, que exprese continuidad a través de saberes y de experiencias útiles sobre la naturaleza y su conservación ecológica (Avendaño, 2012).

Si existieran materias más formales referentes al ambiente en todos los niveles educativos, como sucede en licenciaturas, posgrados y otras carreras relacionadas al medio ambiente, las nuevas generaciones terminarían cada uno de los grados con mayores armas y con un mayor pensamiento crítico para poder enfrentar las problemáticas ambientales que tenemos hoy en día y las que vienen para los próximos años.

Por otra parte, la cultura de este tipo se puede decir que incluye todo aquello que la humanidad ha incorporado en la naturaleza, con el fin de dominarla, transformarla, establecer relaciones sociales acertadas, generar respuesta a sus interrogantes, según lo expresa Beldarrín (2004; citado en Malluk, Delgado y Figueroa, 2016).

En vista de que el tema de la cultura ambiental es tan amplio, es preciso delimitar su estudio. Para Bayón (2006), la cultura ambiental “debe estar sustentada en la relación del hombre con su medio ambiente, y en dicha relación está implícito el conjunto de estilos, costumbres y condiciones de vida de una sociedad con una identidad propia, basada en tradiciones, valores y conocimientos" (pág. 7).

\section{La educación ambiental en los programas de estudio de la SEP}

Dentro de los programas de estudio, la educación ambiental ha formado parte de los contenidos a enseñar, principalmente en los programas que se orientan al área de ciencias; sin embargo, también de forma transversal en otras asignaturas.

Como es sabido por todos, la Constitución Política de los Estados Unidos Mexicanos (Diario Oficial de la Federación [DOF], 29 de enero de 2016) resalta que se debe impartir educación a todos los mexicanos y mexicanas, y aunque no incluye cuestiones ambientales, se deben estudiar los elementos naturales y la preservación, así como la restauración de los equilibrios ecológicos. La educación ambiental, a partir de la década del 2000, se ha integrado a los programas de estudio, si bien no de una manera formal. 


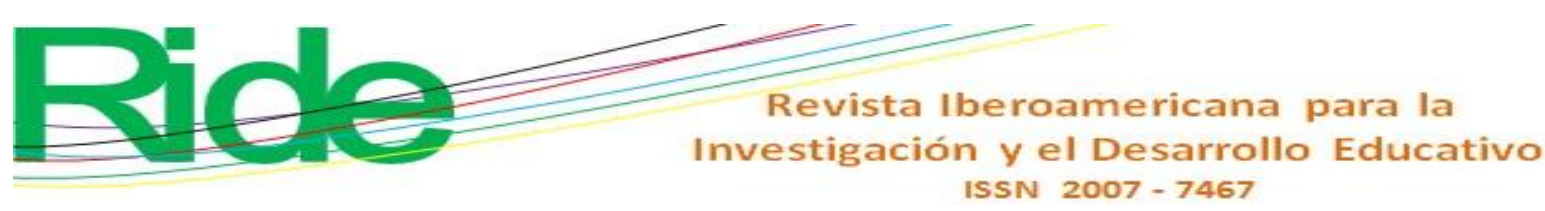

Así, los programas de estudios han incorporado elementos de acuerdo con las tendencias actuales. En una comparativa de los programas de estudios de 1993 y 2009, realizada por la SEP (2009) en el contexto de la reforma integral para la educación básica, se encontró que los cambios a los programas de ciencias naturales obedecen a la consideración de los siguientes aspectos:

- Se incorporan vías de construcción de nociones científicas, que comprende la recuperación y aprovechamiento de las ideas o conocimientos previos como base para la construcción de un número acotado de nociones, conceptos o procesos científicos y el desarrollo de representaciones funcionales, coherentes con las competencias para la vida y el perfil de egreso de la educación básica.

- $\quad$ Se incluye una selección de contenidos de mayores intereses para los alumnos, de acuerdo con sus niveles de comprensión, relevantes, duraderos y aplicables a la resolución de situaciones problemáticas y al contexto social de los estudiantes.

- La experiencia práctica, la intuición, el análisis y la deducción, como una forma de aproximación a nociones científicas

- Destaca la importancia que se le da a fortalecer actitudes de responsabilidad y respeto en los estudiantes, con base en el conocimiento de su cuerpo, y además de reconocer capacidades para asumir iniciativas favorables a su bienestar, vinculadas a la cultura de la prevención y la promoción de la salud.

- $\quad$ Se fortalece la educación ambiental para la sustentabilidad mediante el estudio de temas de interés social: sus componentes natural y social, sus interacciones e impacto en el medio, y la promoción del consumo responsable.

En el caso del Acuerdo 592, se establece que en el área de ciencias a nivel secundaria: "La cultura de la prevención es uno de sus ejes prioritarios, ya que la asignatura favorece la toma de decisiones responsables e informadas en (...) el ambiente; (...) relaciona, a partir de la reflexión, los alcances y límites del conocimiento científico" (SEP, 2011a, p. 30).

En el programa de estudios de Ciencias I, actualmente vigente e impartido en el primer grado de secundaria (SEP, 2011b), dentro del bloque III se encontró que se destaca debido a que se analizan las consecuencias del incremento del efecto invernadero, en términos del calentamiento global y cambio climático. Lo cual da contexto para promover la reflexión en torno a las causas de la contaminación atmosférica y sus efectos en la calidad de vida. 


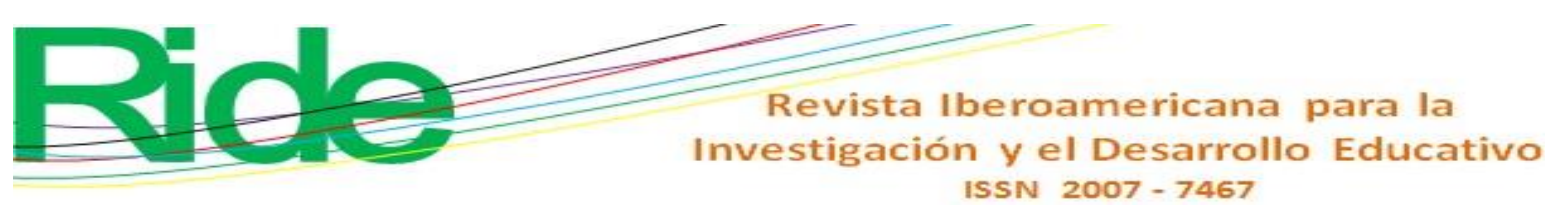

Herrán, 2011, pág. 3). En este sentido, se plantea que las técnicas que utiliza el docente para dirigir la enseñanza tienen un papel predominante en el proceso de enseñanza-aprendizaje porque deben ir en la dirección de facilitar la construcción del conocimiento con sus estudiantes.

En la tabla 1 se muestran algunas de las técnicas recomendadas, con base en de la Herrán (2011), y que sin duda pueden ser fundamentales en la enseñanza de temáticas ambientales.

Tabla 1. Descripción de técnicas de enseñanza

\begin{tabular}{|r|l|}
\hline $\begin{array}{r}\text { Técnica de } \\
\text { enseñanza }\end{array}$ & Descripción \\
\hline $\begin{array}{r}\text { Debate } \\
\text { dirigido }\end{array}$ & $\begin{array}{l}\text { Se utiliza para presentar un contenido y poner en relación los elementos técnicos } \\
\text { presentados en la unidad didáctica con la experiencia de los participantes. El } \\
\text { formador debe hacer preguntas a los participantes para poner en evidencia la } \\
\text { experiencia de ellos y relacionarla con los contenidos técnicos. Durante el } \\
\text { desarrollo de la discusión, el formador puede sintetizar los resultados del debate } \\
\text { bajo la forma de palabras clave para llevar a los participantes a sacar las } \\
\text { conclusiones previstas en el esquema de discusión. }\end{array}$ \\
\hline Lécnica & $\begin{array}{l}\text { Consiste principalmente en la presentación oral de un tema. Su propósito es } \\
\text { transmitir información de un tema, propiciando la comprensión del mismo. Para } \\
\text { ello el docente se auxilia en algunas ocasiones de encuadres fonéticos, ejemplos, } \\
\text { analogías, dictado, preguntas o algún tipo de apoyo visual; todo esto establece } \\
\text { los diversos tipos de exposición que se encuentran presentes y que se abordan a } \\
\text { continuación: exposición con preguntas, en donde se favorecen principalmente } \\
\text { aquellas preguntas de comprensión y que tienen un papel más enfocado a } \\
\text { promover la participación grupal. } \\
\text { ardenando. Esto es casi siempre más productivo que cada persona pensando por } \\
\text { sí sola. }\end{array}$ \\
\hline
\end{tabular}

Fuente: Elaboración propia con información de de la Herrán (2011) 


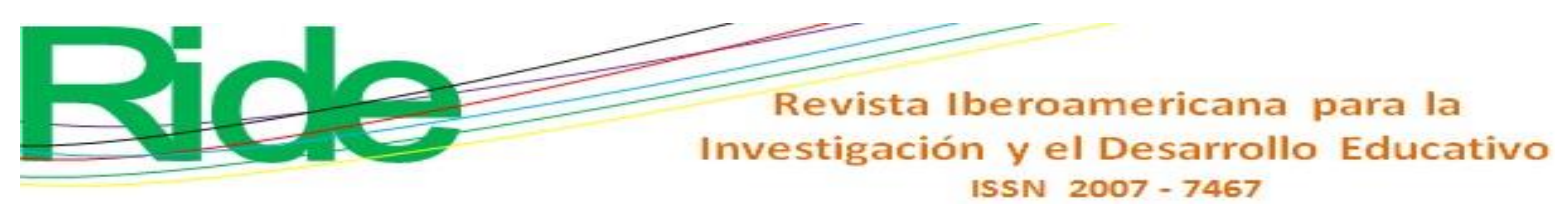

\section{Participantes}

Esta investigación tuvo como participantes al docente y alumno, ya que aquel es el encargado de abordar y presentar las temáticas ambientales y este de modificar su conducta y así crear una cultura ambiental formal. Se aplicó a dos profesores que dan clase de ciencias y a 23 alumnos de primer grado. El método de selección de la muestra fue por conveniencia, pues se presentó el oficio a los directivos de la escuela secundaria y fueron ellos quienes asignaron el grupo y profesor para aplicar los instrumentos.

\section{Instrumento}

El primer instrumento que se aplicó a los profesores constó de 23 preguntas, las cuales fueron de respuesta múltiple, utilizando una escala de Likert de 5 puntos, que iba desde "Muy en desacuerdo" a "Muy de acuerdo". Las preguntas se encaminaron hacia temas que se relacionan con su práctica docente: si utiliza material didáctico para abordar temas ambientales, si aclara las dudas de los alumnos durante y después de abordar los temas, así como si les resalta a sus alumnos la importancia de preservar el planeta.

El segundo instrumento se aplicó a estudiantes, ya que es primordial conocer su opinión respecto a temas referentes al cuidado del ambiente. El cuestionario se conformó de 29 preguntas con respuestas múltiples, al igual que la muestra anterior.

Los dos cuestionarios fueron diseñados por los autores y se validó a partir de juicio de expertos, que es un método de validación útil para verificar la fiabilidad de una investigación y que se define como "una opinión informada de personas con trayectoria en el tema, que son reconocidas por otros como expertos cualificados en este, y que pueden dar información, evidencia, juicios y valoraciones" (Escobar y Cuervo, 2008, p. 29). Los expertos que validaron el instrumento fueron en total cuatro, dos expertos en temas ambientales y dos profesores de secundaria. Las recomendaciones que hicieron se orientaron a la redacción en tres ítems dirigidos a los alumnos, con el objetivo de que se mejorara su entendimiento.

\section{Resultados y discusión}

De acuerdo con los hallazgos encontrados y el análisis de los resultados, los individuos involucrados se están apropiando de la educación ambiental como una herramienta que los lleva a la preservación del medio ambiente. 


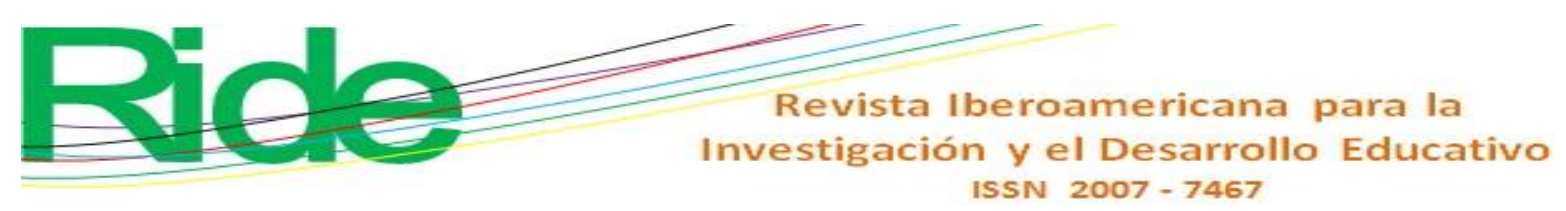

En el caso de los profesores, se les realizaron preguntas sobre su perfil y percepción de los temas ambientales, tal y como se observa en la tabla 2.

Tabla 2. Perfil y percepción de los profesores sobre temas ambientales

\begin{tabular}{|l|c|c|c|c|c|}
\hline Preguntas a profesores & $\mathbf{1}$ & $\mathbf{2}$ & $\mathbf{3}$ & $\mathbf{4}$ & $\mathbf{5}$ \\
\hline $\begin{array}{l}\text { 1. ¿Su perfil de docente está diseñado de acuerdo con programa de estudios } \\
\text { de la materia Ciencias I (Biología)? }\end{array}$ & 0 & 0 & 0 & 0 & 2 \\
\hline $\begin{array}{l}\text { 4. ¿Considera que el programa de estudios de la materia Ciencias I contiene } \\
\text { una buena temática ambiental? }\end{array}$ & 0 & 0 & 0 & 0 & 2 \\
\hline $\begin{array}{l}\text { 6. ¿La institución cuenta con alguna actividad para crear una cultura de } \\
\text { preservación del ambiente? }\end{array}$ & 0 & 0 & 0 & 0 & 2 \\
\hline 10. ¿Le resulta suficiente el tiempo dedicado a los temas ambientales? & 0 & 0 & 0 & 2 & 0 \\
\hline
\end{tabular}

1: Muy en desacuerdo; 2: En desacuerdo; 3: Indiferente; 4: De acuerdo; 5: Muy de acuerdo.

Fuente: Elaboración propia

Con base en las respuestas, los profesores cuentan con el perfil para impartir la asignatura y perciben que el programa y la institución contribuyen a crear una buena cultura de la preservación del ambiente.

Para el caso de las técnicas de enseñanza, las tablas 3 y 4 muestran los resultados de los cuestionamientos de mayor relevancia realizados tanto a maestros como a alumnos. En ellos, las frecuencias muestran una actitud positiva en las preguntas claves, por ejemplo, al cuestionar al alumno y al docente la manera en que abordan estos temas de gran importancia; también se cuestionó si las técnicas de enseñanza que utiliza el docente favorecen al aprendizaje del alumno. Como se puede apreciar en la tabla 3 , los resultados fueron favorables a lo cuestionado. 


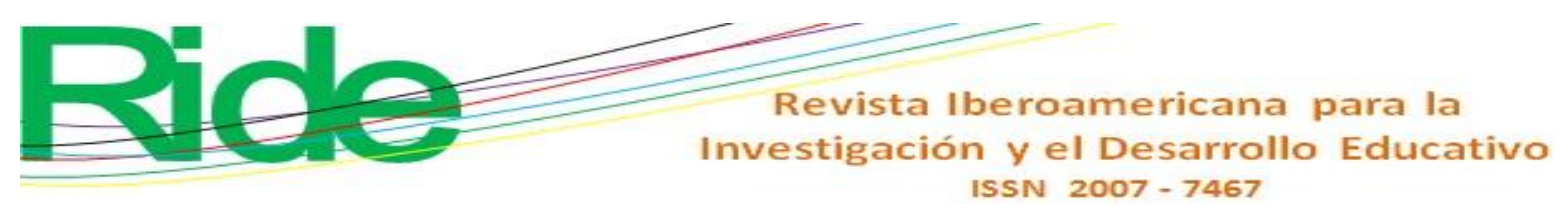

Tabla 4. Técnicas de enseñanza (cuestionario a alumnos)

\begin{tabular}{|l|c|c|c|c|c|}
\hline Preguntas a alumnos & $\mathbf{1}$ & $\mathbf{2}$ & $\mathbf{3}$ & $\mathbf{4}$ & $\mathbf{5}$ \\
\hline 5. Respeto las áreas verdes dentro y fuera de la escuela. & 2 & 1 & 3 & 11 & 11 \\
\hline $\begin{array}{l}\text { 11. iTu profesor hace exposiciones acerca de temas } \\
\text { relacionados con el medio ambiente? }\end{array}$ & 2 & 0 & 4 & 6 & 16 \\
\hline $\begin{array}{l}\text { 13. El profesor aplica actividades de reflexión a los alumnos } \\
\text { para la preservación del medio ambiente. }\end{array}$ & 0 & 2 & 4 & 10 & 12 \\
\hline $\begin{array}{l}\text { 16. iTu profesor utiliza material didáctico (proyector, láminas, } \\
\text { etc. ) para explicar temas ambientales? }\end{array}$ & 3 & 2 & 3 & 10 & 10 \\
\hline $\begin{array}{l}\text { 17. El profesor aplica la técnica de lluvia de ideas en temas } \\
\text { ambientales. }\end{array}$ & 2 & 1 & 1 & 12 & 12 \\
\hline $\begin{array}{l}\text { 24. El profesor aplica actividades en el aula para fomentar el } \\
\text { cuidado del medio ambiente. }\end{array}$ & 2 & 4 & 2 & 9 & 11 \\
\hline $\begin{array}{l}\text { 27. El profesor fomenta el debate como estrategia de } \\
\text { aprendizaje. }\end{array}$ & 2 & 4 & 5 & 7 & 10 \\
\hline
\end{tabular}

Fuente: Elaboración propia

A partir de lo anterior se puede inferir que el nivel de cumplimiento de los objetivos planteados al principio de la investigación fue alto, ya que, después de realizar un análisis de los objetivos planteados y revisar los resultados de ambas muestras que se aplicaron, los resultados demuestran que las técnicas de enseñanza que está utilizando el docente para impartir temáticas ambientales están siendo las adecuadas, y que están favoreciendo al estudiante a crear un pensamiento crítico y reflexivo acerca de la situación actual del planeta. Esto conlleva a que los jóvenes desarrollen una cultura de preservación al mismo tiempo que se ve modificada su conducta.

Únicamente a través de la educación es la manera en que el individuo interioriza la cultura para ser capaz de construir conocimientos y a su vez modificar sus acciones y contribuir como sujeto individual a la transformación de la realidad del medio ambiente (Ferrer, Menéndez y Gutiérrez, 2004).

En el caso de los estudiantes, cuando se les preguntó si el profesor explica a los estudiantes la importancia de cuidar el medio ambiente, $50 \%$ señaló estar muy de acuerdo, y solo $7.1 \%$ respondió lo contrario. Para mejorar aún más este aspecto el docente tiene que hacer mayor énfasis en la importancia de cuidar la Tierra. Ya que la preservación del medio ambiente implica respetar la naturaleza, hacer buen uso del agua, practicar el reciclaje; de no hacerlo, las consecuencias son

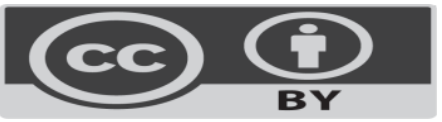




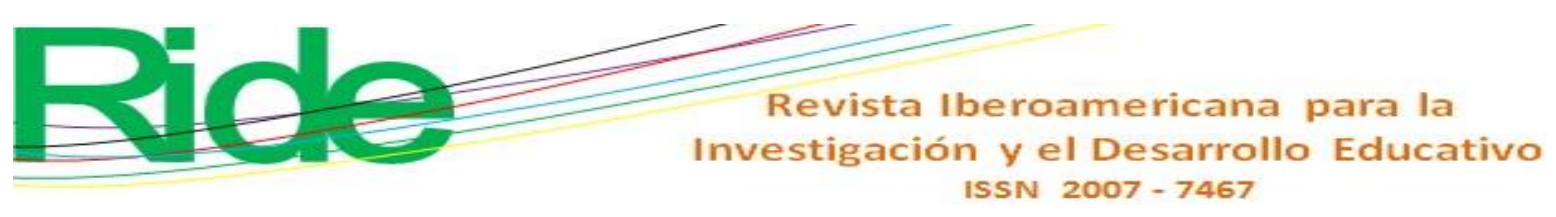

graves: aumentarían las enfermedades respiratorias, la demanda del agua potable y las temperaturas, esto último, a su vez, permitirá la reproducción de ciertos insectos que causarán enfermedades a las plantas y afectarán los cultivos, por mencionar tan solo un ejemplo.

En el caso de la pregunta sobre si su profesor fomenta actividades de reflexión acerca del cuidado del medio ambiente, 42.9 \% de los alumnos respondieron la opción "Muy de acuerdo". Esta es una de las preguntas más importantes en la encuesta, ya que el que el alumno practique actividades de reflexión lo ayudarán a modificar su conducta y ser más consciente de los problemas ambientales actuales. De esta forma, coinciden en que el camino mediante el cual el profesor los lleva a la reflexión es a través de técnicas como el debate dirigido, donde los alumnos se informan sobre alguna problemática, debaten sobre su importancia y reflexionan sobre su forma de actuar.

Al cuestionarle al alumnado si su profesor o profesora fomenta algunas de las técnicas de enseñanza ya mencionadas, los resultados apuntan a que sí se ponen en práctica. Que sean favorables los resultados de estas preguntas indica que este tipo de técnicas implementadas por el docente son propositivas para que el estudiante desarrolle la formación de un pensamiento crítico y reflexivo acerca del cuidado del medio ambiente.

Por parte del docente, también se le cuestionó si utiliza dichas técnicas de enseñanza, el resultado de las encuestas apunta a que sí están siendo puestas en práctica. Es importante implementar las diversas técnicas de enseñanza debido a que se encuentran en constante relación con las características personales y habilidades profesionales del docente. Como se mencionó en unos de los apartados anteriores, esto ayuda a que el estudiante desarrolle de una manera más adecuada sus aprendizajes y a mejorar su comportamiento con el medio ambiente. Finalmente, al cuestionarle al alumno acerca de la forma en cómo imparte la clase su docente, los porcentajes indican que este último les explica cuando ellos, los estudiante, no tienen información clara, y que sí resuelve las dudas que se presentan al concluir algún tema. Estos datos fueron arrojados en en los ítems 19, 23, 26. Que el docente resuelva las dudas de sus alumnos nos habla del conocimiento que tiene de los contenidos. 


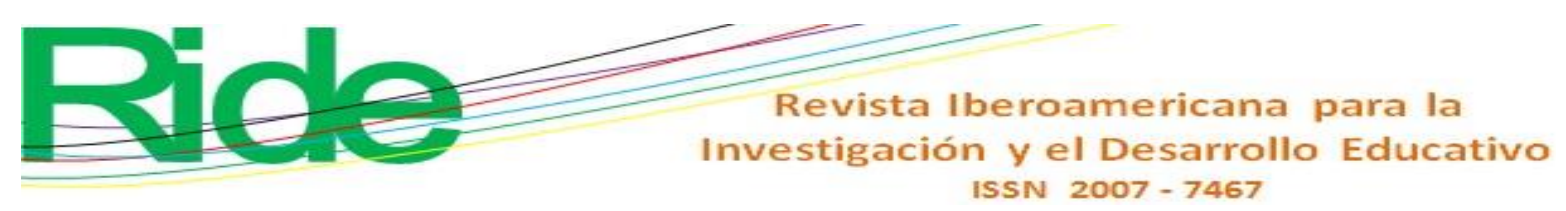

\section{Conclusiones}

La conservación ambiental debe ir ligada a una adecuada educación ambiental, dado que es un proceso que pretende potenciar actitudes de conservación y mejoramiento del ambiente que conduzcan a crear una cultura de preservación. El docente tiene como tarea seguir desarrollando estrategias en busca de transformar la cultura de sus estudiantes, ya que tener aprendizajes continuos acerca de esta temática conducirá a un futuro mejor para las siguientes generaciones.

Las instituciones, al igual que el docente, juegan un papel importante en la educación ambiental de sus estudiantes. El docente como guía y facilitador del aprendizaje, por medio de la actualización constante de los temas ambientales, así como el mejoramiento de las técnicas de enseñanza que utiliza a diario con sus alumnos, pues estas son una herramienta fundamental para el desarrollo de la cultura ambiental. Es necesario igualmente que busque la manera de abordar las dudas que queden al término de cada tema, porque de esta manera le será más fácil concebir el aprendizaje al alumno. La institución, por su parte, debe de tener la iniciativa de implementar nuevos programas, actividades y acciones para favorecer el cuidado del planeta. Por ejemplo, diseñar métodos para el ahorro del agua, para el ahorro de energía, control de residuos dentro y fuera de la institución, e involucrar a padres de familia y habitantes de la comunidad a replicar este tipo de acciones.

La implementación de estrategias docentes no solo pueden ser dentro del aula, sino también fuera de ella, tal es el caso del fomento de la participación y vinculación de la misma comunidad educativa con apoyo de directivos. Esto ayudará a estar en un mismo canal; por ejemplo, una manera eficaz de aprendizaje es promover actividad lúdicas por medio de talleres o concursos enfocados en la importancia de diferentes factores ambientales. La competencia genera esa característica propia del individuo de aprovechar la información y trabajar en equipo para alcanzar una meta. Este tipo de actividad contribuirá a generar una conciencia ambiental.

Existen varias maneras de alcanzar los objetivos trazados para preservar el ambiente. El reto es buscar la manera más adecuada de lograrlo. Sabemos que una de las mejores armas es la educación. El docente tiene como tarea estar actualizado en cuanto a diseñar nuevas estrategias de enseñanza debido a que los tiempos cambian y las nuevas generaciones de estudiantes demandan nuevas formas de aprendizaje. En este sentido, este trabajo puede servir de guía para futuras investigaciones que podrían enfocarse en la enseñanza. Se recomendaría realizar una observación para verificar que las técnicas a las que aluden los profesores sean las que se realizan en el aula, o bien, observar a los alumnos y cotejar que su participación en el cuidado del ambiente ha mejorado 


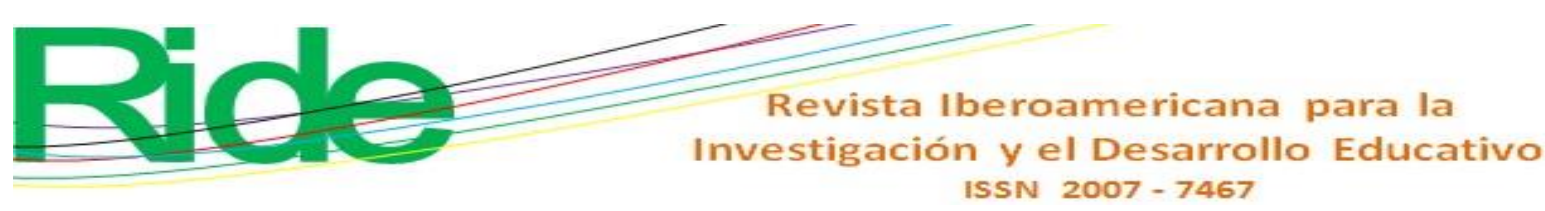

con la implementación de dichos métodos de enseñanza. Asimismo, se podrían orientar en analizar la articulación y secuencia de las técnicas de enseñanza que se utilizan a lo largo de la educación básica para conocer si tienen un impacto en el egresado de la misma.

\section{Referencias}

Avendaño, W. (2012). La educacion ambiental (EA) como herramienta de la responsabilidad social (RS). Revista Luna Azul, (35), 94-115. Recuperado de https://www.redalyc.org/pdf/3217/321727349006.pdf.

Bayón, P. (2006). Educación ambiental, participación y transformación social sostenible en Cuba. Revista Interface, 2(4), 89-104. Recuperado de http://biblioteca.filosofia.cu/php/export.php?format=htm\&id=2335\&view=1.

Calixto, R. (2015). Educación Ambiental para la Sustentabilidad en la Educación Secundaria. Revista Actualidades Investigativas en Educación, 15 (3). Universidad de Costa Rica: Costa Rica.

De La Herrán, A. (2011). Técnicas didácticas para una enseñanza más formativa. En Álvarez, N. y Cardoso, R. (coords.), Estrategias y metodologías para la formación del estudiante en la actualidad. Camagüey, Cuba: Universidad de Camagüey. Recuperado de http://radicaleinclusiva.com/wp-content/uploads/2018/01/teuniv.pdf.

Diario Oficial de la Federación [DOF]. (29 de enero de 2016). Constitución Política de los Estados Unidos Mexicanos. Diario Oficial de la Federación. Recuperado de www.ordenjuridico.gob.mx/Constitucion/cn16.

Escobar, J. y Cuervo, A. (2008). Validez de contenido y juicio de expertos: una aproximación a su utilización. Avances en Medición, 6, 27-36. Recuperado de http://www.humanas.unal.edu.co/psicometria/files/7113/8574/5708/Articulo3_Juicio_de_ expertos_27-36.pdf.

Ferrer, B., Menéndez, L. y Gutiérrez, M. (2004). La cultura ambiental por un desarrollo sano y sostenible. La experiencia de Cayo Granma. Santiago, (104). Recuperado de http://cursos.clavijero.edu.mx/cursos/191_gdpf/modulo3/tareas/documentos/Estudio_de_c aso_3.pdf.

Hernández, R, Fernández, C, Baptista, P. (2010) Metodología de la investigación (5. ${ }^{a}$ ed.). Ciudad de México, México: McGraw Hill. 


\section{Revista Iberoamericana para la Investigación y el Desarrollo Educativo

\begin{tabular}{|c|c|}
\hline Rol de Contribución & Autor (es) \\
\hline Conceptualización & Martin/ Rosa / Xochitl «igual» \\
\hline Metodología & Martín /Rosa «igual» \\
\hline Software & Martín Olguín / XócHITL «igual» \\
\hline Validación & Martín Olguín «que apoya» / XÓCHITL «principal» \\
\hline Análisis Formal & Martín Olguín«que apoya» / XÓCHITL «principal» \\
\hline Investigación & Martin «que apoya» / Rosa «principal» \\
\hline Recursos & Martin / Rosa / Xochitl «igual» \\
\hline Curación de datos & Martín Olguín «que apoya» / XóCHITL «principal» \\
\hline $\begin{array}{l}\text { Escritura - Preparación del borrador } \\
\text { original }\end{array}$ & Martin «que apoya» / Rosa«principal» / Xochit|«principal» \\
\hline Escritura - Revisión y edición & Martin «que apoya» / Rosa«principal» / Xochit|«principal» \\
\hline Visualización & Martin / Rosa/ Xochitl «igual» \\
\hline Supervisión & ROSA «principal» \\
\hline Administración de Proyectos & ROSA/XOCHITL «igual» \\
\hline Adquisición de fondos & NO APLICA \\
\hline
\end{tabular}

